\title{
Frontières
}

\section{Une tombe pas ordinaire}

\section{Maurice Boutin}

Volume 18, numéro 2, printemps 2006

La mort dans tous ses états

URI : https://id.erudit.org/iderudit/1073218ar

DOI : https://doi.org/10.7202/1073218ar

Aller au sommaire du numéro

Éditeur(s)

Université du Québec à Montréal

ISSN

1180-3479 (imprimé)

1916-0976 (numérique)

Découvrir la revue

\section{Citer cet article}

Boutin, M. (2006). Une tombe pas ordinaire. Frontières, 18(2), 34-36. https://doi.org/10.7202/1073218ar

\section{Résumé de l'article}

Edmond Jabès entend maintenir une distance capable de transformer en promesse ce qu'elle rend possible : non pas une identité, mais cette quête du lieu qu'est le Livre, dans un renouveau radical de la théophanie à même l'intérêt majeur pour l'écriture dans la modernité. d'utilisation que vous pouvez consulter en ligne.

https://apropos.erudit.org/fr/usagers/politique-dutilisation/ 


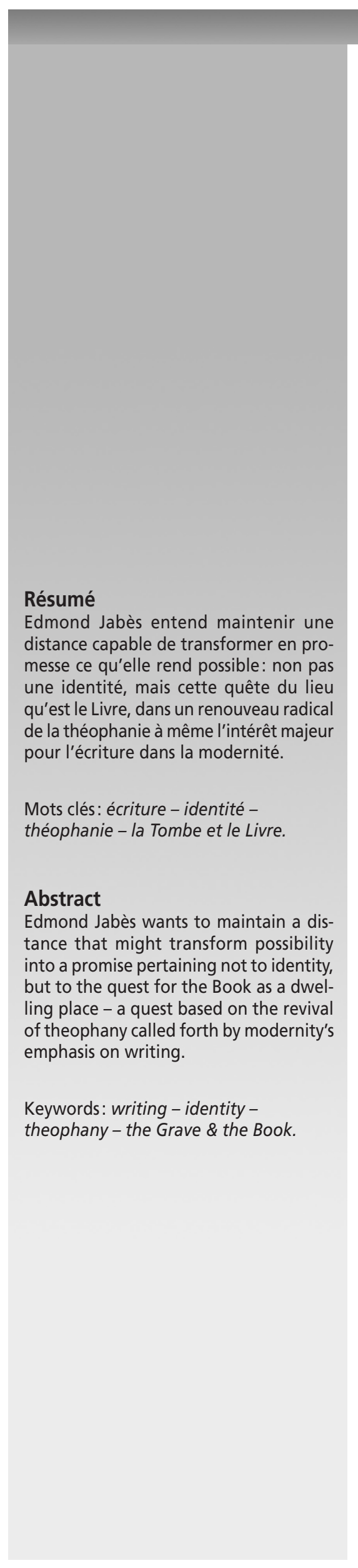

T I C $\quad$ L $\quad E$

\section{UNE TOMBE PAS ORDINAIRE}

\author{
Maurice Boutin, Ph. D., \\ titulaire, Chaire J.W. McConnell de philosophie \\ de la religion, Université McGill.
}

L'obscurité dans laquelle toute personne est plongée par le décès ne fait place - au mieux! - qu'à une histoire personnelle qui n'est plus vécue mais seulement racontée par d'autres comme pour être tirée de l'oubli. Ce rapport de dépendance envers une parole d'autrui qui me concerne en mon absence se retrouve aussi dans l'écriture, qui dissimule pudiquement une nostalgie dans l'attente de l'inimaginable: le récit, qui reste à espérer pour (continuer à) vivre.

La voix du poète Edmond Jabès, on ne l'entend plus depuis 1991; mais elle continue de parler à travers tous ces rabbins imaginaires qui étoilent son œuvre et ne scintillent que sur fond de nuit et parce qu'il y a la nuit. Rabbins imaginaires plus en dialogue avec le langage qu'entre eux, tout adonnés qu'ils sont à une même tâche: maintenir et ranimer une attention extrême à cette écoute que le langage est à lui-même, dans la solitude et le désarroi des vocables abandonnés, laissés à eux-mêmes.

Laisser les vocables à eux-mêmes, c'est les entendre converser entre eux. C'est aussi aborder l'indicible d'une équivoque propre à nourrir un in-sensé désir : celui de congédier la nette séparation du ciel et de la terre, de la terre et du ciel.

C'est dans le refus de cette séparation que s'enracine la poésie de Jabès. Un enracinement qui ne laisse d'autres traces que celles dessinées par le mouvement des mots entre eux, comme le sable du désert permet le libre jeu du vent sur les dunes.

\section{UNE PAROLE DE SABLE}

Né au Caire en Égypte en 1912, Jabès est resté profondément marqué par la réalité du désert. Il s'en explique dans ses entretiens avec Marcel Cohen publiés en 1981 sous le titre: Du désert au livre ${ }^{1}$. Dans les dialogues de Jabès, et grâce à eux, les mots coulent, "étourdis de l'immense souffle» du vent (LM 171), aussi insaisissables que le sable qui coule entre les doigts. Le langage s'écoule plus qu'il ne s'échange; il s'écoule, mais sans s'épuiser, abordant aux hautes rives du livre. "J'étais, dit Jabès, sans le savoir, à l'écoute d'un livre qui rejetait tous les livres et que, bien évidemment, je ne maîtrisais pas. Un livre que j'interrogeais en même temps que je l'écrivais et dont j'attendais qu'il se fît à travers cette interrogation même " (LM 171). "Si j'avais à définir la parole de mes livres, je dirais qu'elle est parole des sables - de sable - un bref instant audible, visible: parole d'une écoute extrême et d'une mémoire très ancienne » (LM 180).

Capter la parole? Non pas: comment?, mais : quand? Au moment, éblouissant, de son surgissement, ou au moment, insensible, de son évanouissement? (LM 180) Jabès est fasciné par cet évanouissement, alors que la parole rejoint dans sa chute le néant qu'elle illumine en émergeant. Le mystère, pour Jabès, n'est pas «dans les êtres ou dans les choses; mais au bout, là où ils ne sont plus rien » (LM 191). Ce bout n'est pas un terme; c'est une borne. 

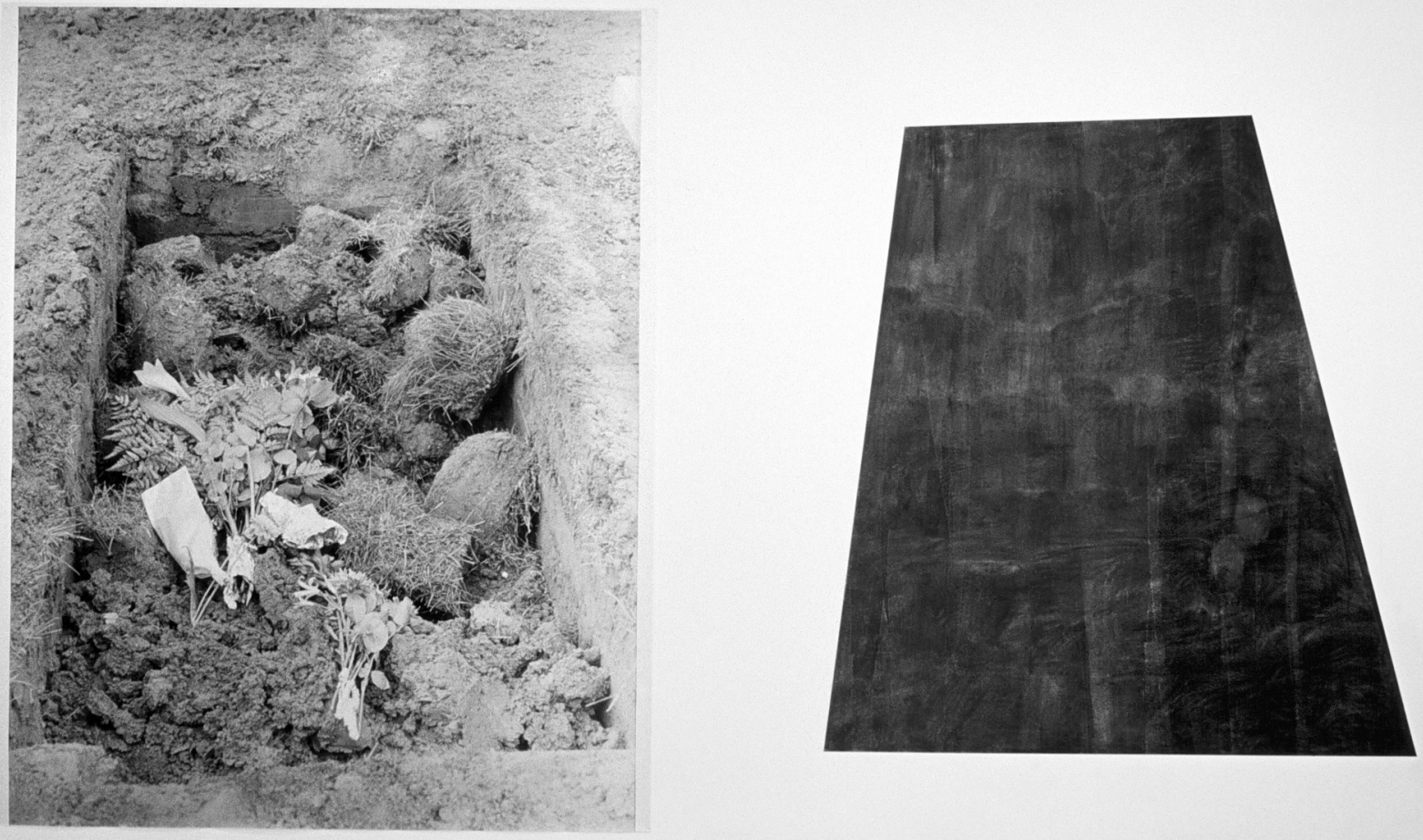

Secret mouvement de la distance qui, dans la lettre, affecte le vocable et de là tout ce qui doit au vocable d'exister. La distance est interne au vocable et partant, intérieure à l'être humain qui ne s'unit à soi qu'en s'opposant à soi, qui se perd en soi, pur vocable, signe séparé. Car dans le vocable s'opère une séparation que chaque lettre accentue en l'annulant (LM 114).

La question n'est pas d'investir la distance séparant le mot de ce qu'il désigne (DL 77), mais d'entendre, dans le mot, sa tension vers le neutre, le vide, sa manière à lui d'explorer le néant. C'est cela, écouter le texte (DL 78) : le saisir dans sa réalité, «là où les mots sont confrontés à l'infini qui les mine $»(\mathrm{DL} 80)$.

\section{PROMESSE SANS TÉMOIN}

La révélation de son destin profond, comme Jabès le dit à Marcel Cohen en 1981, est confirmation du destin collectif juif (DL 48). Transformer en promesse ce que ce destin rend possible, c'est avouer que l'événement de cette révélation nous dépasse.

Révélation et promesse excluent le témoignage (LM 27), parce que «la durée

LE MONDE EST DE POUSSIÈRE. IL REDOUTE LE VENT.

ET POURTANT, SEUL LE SOUFFLE DE LA PAROLE PEUT TRACER EN LUI

\section{LES CONTOURS DU RÉEL, LES MOTS QUI DISENT LA VIE INCERTAINE.}

ne nous appartient pas» (LM 180). Jabès «ne cherche pas à être le témoin. Il est seulement à l'écoute des mots qui tracent son avenir» (DL 54). Seule la parole témoigne du trajet parcouru par elle (LM 180). C'est le livre qui témoigne... De quoi ? D'un conflit: celui d'une «parole immortelle opposée à la parole de toute finitude» et «qu'aucune page du livre ne saurait résoudre» (LM 183). Ce sont les mots, le livre, et eux seuls qui témoignent de la naissance de toute chose (DL 121). Et le mystère est dans le vocable.

Penser ensemble révélation et promesse (LM 50) ne dispense pourtant pas d'éprouver aussi la révélation comme promesse, et toute promesse comme une révélation de ce qui est. La mise en mots de l'espérance (LM 95) est ce qui demeure: le futur. Non le présent, qui désole (LM 159; DL 5l).

\section{L'IDENTITÉ ET SA DÉ-ROUTE}

$\mathrm{Ne}$ plus se demander ce qu'on fait là, comment on y est parvenu, par quels détours. Écouter plutôt... (LNM 64). La poésie de Jabès déroute parce qu'elle ignore la question: qui suis-je? «La question: qui suis-je? n'a, pour moi, aucun sens. Elle n'a jamais effleuré mon esprit. Peut-être parce que l'identité qui n'est que le besoin légitime d'avoir un visage à exhiber n'est, en fait, que le désir, condamné à rester à l'état de désir, d'une affirmation de nousmêmes constamment différée» (LM 185). Être, c'est assumer un au-delà (LM 185); et être hanté par la question de l'identité, c'est rester pris dans l'anonymat du délai.

L'identité est le nom. Quatre lettres ont suffi pour que Dieu soit Dieu. Pour l'homme, il en a fallu cinq, dont une double. Qu'est-ce que cela veut dire? Eh bien, cela signifie tout simplement 
que le langage nous prive d'identité en nous en offrant une qui n'est qu'un assemblage de lettres n'appartenant qu'à lui et que nous retrouvons dispersées un peu partout. La lettre est anonyme. Elle est un son et un signe. En participant à la formation du nom, elle crée, à travers lui, notre image. Elle cesse, alors, d'être anonyme pour faire corps avec nous. Elle épouse notre condition ou notre incondition, vit et meurt de notre vie et de notre mort. Qui nous interpelle, l'interpelle d'abord. Mais est-ce de la lettre ou de son reflet qu'il s'agit? De son reflet, sans doute. En ce cas, notre nom ne serait que le reflet d'une absence de nom que cette absence même aurait composé. D'où notre absence au monde dont notre nom répond; d'où notre présence à l'être absent dont nous avons hérité du nom. La lettre est à l'être ce que la mémoire est à l'oubli : à la fois le déroulement de son histoire et le sceau de son éternel sommeil (DL 18-19).

Il y a donc bien une réponse à la question: qui suis-je? Mais cette réponse n'est que question puisque «si je suis la trace, je ne puis l'être que pour l'autre; mais si l'autre est autrui, autre de l'autre, qui relèvera la trace» que je suis pour autrui et qu'autrui est pour moi en tant qu'autre? Si je suis la trace, autrui est, peut-être, l'abîme de la trace (LM 169). Si je suis la trace, alors mon identité, quelle est-elle? Une " pauvre plaque d'identité sous le talon» (LM 78).

Pour Jabès, l'identité n'est plus une question (DL 99), mais deux: la question de l'autre dans la question de soi, soi séparé de soi, en distance, par l'autre, de soi. Être, c'est devenir (DL 113) ; et l'identité, c'est ce qu'on choisit d'être en devenant (DL 100). «Nous ne sommes que l'idée que nous nous faisons de nous-mêmes » (DL 76), et « cette idée est un leurre, un mensonge dès qu'on la fige, dès qu'on la donne pour exemplaire» (DL 111).

\section{LA QUÊTE DU LIEU ET LE LIVRE}

Dans les esquisses d'une éthique de l'autre tracées à même la quête du lieu, on ne saurait faire la part de Dieu, sinon pour rappeler que «Dieu est le lieu», «qui est la perte du nôtre» (LM 26). «Dieu est le lieu - comme le livre»(DL 35). Jabès l'avoue, «ce rapprochement m'a toujours excité. Dieu, à travers Son Nom, est le livre. C'est pourquoi [...] l'on n'écrit que dans l'effacement du Nom divin - du lieu » (DL 35), au-delà de la parole (DL 143).

Jabès est fasciné par l'inquiétude entretenue dans le langage par l'irruption du mot «Dieu». Mot qui interrompt le jeu réglé de la syntaxe; mot in-sensé, qui émancipe les signes, qui disperse le sens, fait pour proliférer et non pour se réfugier dans un mot figé et fixe, donneur de certitude - et donc de mort. Mes livres, dit Jabès, «ne deviennent illisibles que si l'on y cherche une certitude» (DL 158). Dans la poésie de Jabès, Dieu est restitué à sa réalité de mot. Ce qui n'est pas rien quand le langage est remis à lui-même, se met à s'écouter lui-même, à creuser en lui cette distance vertigineuse qui le sépare de lui-même.

Le mot «Dieu» dans le langage, c'est comme le livre ouvert sur la table: il occupe bien peu de place, et pourtant, l'espace qu'il investit est immense (DL 50). Cela tient de l'infinie résonance des quatre lettres de ce mot. Ce n'est plus tant le buisson ardent qui, comme le rapporte la Bible au livre de l'Exode (chapitre 3, verset 2), brûle sans se consumer; ce sont les quatre incendies de ces quatre lettres (LM 43) qui font du livre de Dieu le nouveau désert de l'humain. Dans cette poignée de sable ramassé dans le désert que sont les mots du langage, on ne distrait point un grain pour le peser; le mot «Dieu» est empoigné avec les autres vocables.

Le monde est de poussière. Il redoute le vent. Et pourtant, seul le souffle de la parole peut tracer en lui les contours du réel, les mots qui disent la vie incertaine. Edmond Jabès s'est tu. Mais les rabbins imaginaires qui peuplent sa poésie se font encore entendre jusque dans ce qu'ils laissent informulé (LM 87). Pour eux, les paroles suivantes de Jabès sont comme un testament: "Je suis tous les autres que je serai. Je ne serai pas. Ils seront moi qui ne puis être» (LM 173).

\section{LE LIVRE, UNE TOMBE}

La poésie de Jabès permet de déceler certaines composantes de la modernité et de découvrir la nature des discontinuités que la modernité permet de mettre en œuvre de manière parfois très surprenante. En effet, Jabès prend acte de l'importance et du rôle qu'on accorde à l'écriture aujourd'hui, mais pour en faire l'occasion par excellence du renouvellement d'une question dont le traitement était demeuré inchangé depuis près de trois mille ans : la question des manifestations de Dieu qu'on appelle habituellement des théophanies. Ce qui retient tout particulièrement l'attention de Jabès, c'est la manifestation de Yahvé qui révèle son nom à Moïse dans l'épisode biblique relaté au livre de l'Exode qui parle du buisson qui brûle sans se consumer. Pour Jabès, l'écriture est aujourd'hui le lieu nouveau et premier de la théophanie. Dans l'écriture, Dieu se distancie de Dieu, et l'être humain se distancie de soi. Unis dans la même aventure de l'écriture, l'écriture les sépare aussi, et surtout elle les sépare d'eux-mêmes.
Dieu ne se souvenant de rien - pas même de son nom ${ }^{2}$, tout entier à son geste d'écriture, écrit sur un rectangle d'air découpé dans le vide ${ }^{3}$, trace ses lettres dans l'avenir toujours sans trace (LM 102). Dieu se retire dans l'écriture; c'est en cela qu'est son infinité. C'est que Dieu aussi est occupé à composer son livre, là, de l'autre côté de ma table. La flamme de ma chandelle lui sert de plume (LM 44), et son livre m'enveloppe de fumée. "Que sera bientôt mon livre sinon un peu de cendre sur une des pages du sien?»(LM 144).

«Le livre n'est pas une tombe ordinaire» (LM 144).

\section{Notes}

1. Belfond, Paris,1981, 173 p. Les abréviations utilisées se rapportent aux livres suivants d'Edmond Jabès: $\mathrm{DL}=D u$ désert au livre. $\mathrm{LM}=$ Le livre des marges (1975 et 1984) [Le livre de Poche - Biblio essais, 4063], Fata Morgana, Paris, 1987, 219 p.

2. Ce qui suit s'inspire surtout de E. Jabès, Ça suit son cours, dans LM; voir surtout LM 44, 73, 102, 144, 173, 183

3. LM 173. Ces mots de Jabès peuvent être comparés à la remarque suivante de Walter Mossberg dans The Wall Street Journal, reproduite dans la publicité du New iMac publiée dans le magazine Newsweek du 27 mai 2002, p. 3: "When you're actually working on the new iMac with the screen lowered so it sits between your face and the base, it's a thing of pure beauty. You feel as if you're typing onto a gorgeous palette that's floating in the air » («Lorsque vous utilisez le nouveau iMac et que vous abaissez l'écran de sorte qu'il se trouve entre votre visage et le clavier, c'est une pure beauté. C'est comme si vous écriviez sur une merveilleuse tablette qui flotte dans les airs. ») 